\title{
Multiple Beam Lattice Imaging of Hexagonal Ferrites
}

\author{
Yoshihiko Hirotsu*, Yoshio Nakamura**, Junnosuke Mizutani*, \\ Sigemaro Nagakura** and Tadahisa Nakamura*
}

\begin{abstract}
Multiple-beam electron microscope lattice imaging was found to be applicable to the direct analysis of crystal structures and defects in hexagonal ferrites. Numerical calculations based on the multi-slice method were made to find an optimum imaging condition suitable for the imaging of barium atom positions in hexagonal ferrites. It was shown that under the condition of nearly fixed under-focus barium atom positions are visible as bright spots in a relatively wide range of crystal thickness. The condition for the imaging was checked experimentally by the observations of $M$ and $Y$ compounds, and the imaging method was used for the analysis of the compositional faults introduced in the $\mathbf{M}$ compound. The origin of the interpretable images with the bright spot contrasts was discussed on the basis of the dynamical effect of electron scattering.
\end{abstract}

(Received December 16, 1982)

Keyzerds: lattice imaging, hexagonal ferrites, structure determination, intergrowth structure, high resolution electron microscopy

\section{Introduction}

A number of structural modifications with hexagonal or rhombohedral long period structures are found in the $\mathrm{BaO}-\mathrm{MeO}-\mathrm{Fe}_{2} \mathrm{O}_{3}$ system $^{(1)-(3)}$, where $\mathrm{Me}$ stands for a divalent spinel-forming metal element $(\mathrm{Fe}, \mathrm{Zn}$, Co or Ni). These compounds, called hexagonal ferrites, are formed by the ordered stacking of invariant structural building units along the $c$-axis. These building units are called $\mathrm{S}, \mathbf{R}$ and $\mathrm{T}$ blocks, which are illustrated in Fig. 1. These blocks have a common cross sectional dimension $a=0.588 \mathrm{~nm}$ in $c$-plane and are characterized by the oxygen frameworks having two (S), three (R) and four (T) oxygen layers. In the middle oxygen layers in $\mathrm{R}$ and $\mathrm{T}$ blocks, every fourth oxygen atom is replaced by a barium atom to form single (R) and double $(T)$ barium-oxygen $(\mathrm{Ba}-\mathrm{O})$ layers with six and threefold symmetry along the $c$-axis, respectively. $\mathrm{S}$ has two oxygen layers and has an atomic arrangement of spinel structure. In these blocks, $\mathrm{Me}$ and $\mathrm{Fe}$ atoms are located in the interstitial sites formed by the oxygen atoms.

$\mathrm{M}\left(\mathrm{BaO} \cdot 6 \mathrm{Fe}_{2} \mathrm{O}_{3}\right)$ and $\mathrm{Y}(2 \mathrm{BaO} \cdot 2 \mathrm{MeO}$.

* Technological University of Nagaoka, Nagaoka 949-54, Japan.

** Tokyo Institute of Technology, Meguro-ku, Tokyo 152, Japan.
$6 \mathrm{Fe}_{2} \mathrm{O}_{3}$ ) are well-known compounds useful for the magnetic materials. Several examples of the stacking sequences and the $c$-parameters of hexagonal ferrites are shown in Table 1.
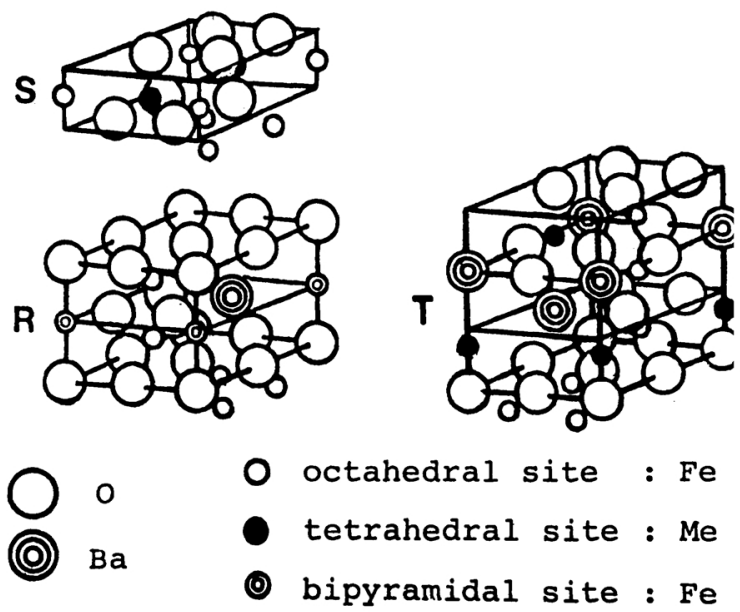

Fig. 1 Perspective drawings of the three fundamental building units: $\mathbf{R}, \mathrm{T}$ and $\mathrm{S}$ blocks.

Table 1 Some examples of the structural series.

\begin{tabular}{llc}
\hline \multicolumn{1}{c}{ Compound } & $\begin{array}{l}\text { Structural } \\
\text { sequence }\end{array}$ & $\begin{array}{c}c \text { parameter } \\
(\mathrm{nm})\end{array}$ \\
\hline $\mathrm{BaFe}_{12} \mathrm{O}_{19}(\mathrm{M})$ & $(\mathrm{RS})_{2}$ & 2.32 \\
$\mathrm{BaMe}_{2} \mathrm{Fe}_{12} \mathrm{O}_{22}(\mathrm{Y})$ & $(\mathrm{TS})_{3}$ & 4.36 \\
$\mathrm{BaMe}_{2} \mathrm{Fe}_{16} \mathrm{O}_{27}(\mathrm{~W})$ & $(\mathrm{RSS})_{2}$ & 3.29 \\
$\mathrm{Ba}_{3} \mathrm{Me}_{2} \mathrm{Fe}_{24} \mathrm{O}_{41}(\mathrm{Z})$ & (RSTS) & 5.23 \\
$\mathrm{Ba}_{2} \mathrm{Me}_{2} \mathrm{Fe}_{28} \mathrm{O}_{46}(\mathrm{X})$ & (RSRS) & 8.41 \\
$\mathrm{Ba}_{4} \mathrm{Me}_{2} \mathrm{Fe}_{36} \mathrm{O}_{60}(\mathrm{U})$ & (RSRSTS) & 11.32 \\
\hline
\end{tabular}


When one of the phases of hexagonal ferrites is grown, the neighboring phases tend to intergrow in several unit cell widths with a common growth axis ( $c$-axis). Such an intergrowth, called microsyntactic intergrowth, has been detected by TEM lattice imaging ${ }^{(4)-(7)}$, together with defect structures intergrown in the form of isolated structural units like $R, S$, RS, RSS, RSSS, $\cdots$. These intergrown structures are ascribed to the structural compensation of the local composition fluctuation ${ }^{(6)(7)}$.

As shown in Fig. 1, S, R and T blocks are characterized by the numbers of $\mathrm{Ba}-\mathrm{O}$ layers; zero(S), one(R) and two(T). Therefore, if $\mathrm{Ba}$ atom positions in hexagonal ferrites are imaged by the high resolution "structure imaging" method, we immediately realize the stacking modes of the building blocks and can determine directly not only the crystal structures, but also details of the microsyntactic intergrowth and defects introduced by the composition fluctuation.

This paper is concerned with the method of the direct analysis of crystal structure and defects by the imaging of barium atom positions. Details of the image calculations to find the optimum condition for the imaging are first reported followed by some examples of the direct analysis.

\section{Image Calculations}

Recently, one and two dimensional long period $^{(8)(9)}$ and antiphase structures ${ }^{(10)(11)}$ of alloys have been analysed in detail on the bases of the direct imaging of atoms by using highvoltage, high-resolution electron microscopy. In each of their observations, ordered bright dot contrasts were imaged in a rather thicker part of the specimens nearly under the Scherzer focus condition ${ }^{(13)}$, corresponding to the positions of atoms occupied substitutionally in the base crystal lattice to form the superstructure. Based on the image calculations, it was confirmed that these images are contributed predominantly by the super-lattice reflections ${ }^{(8)(9)(12)}$. This kind of image is called a "super-structure image".

In the present study, we have tried to image the barium atom positions in hexagonal fer-
Table 2 Electron optical parameters.

\begin{tabular}{lc}
\hline \hline Electron wave length (nm) & $2.51 \times 10^{-3}$ \\
Spherical aberration constant (mm) & 1.2 \\
Incident beam divergence (rad) & $1.0 \times 10^{-3}$ \\
Defocus due to chromatic aberration (nm) & 7.5 \\
Objective aperture radius $\left(\mathrm{nm}^{-1}\right)$ & 5.4 \\
Crystal thickness for one slice (nm) & 0.51 \\
Number of beams excited: & \\
$\quad$ M compound & 1003 \\
Y compound & 1241 \\
\hline
\end{tabular}

rites in a rather thin part of the specimens by choosing the focus condition suitable for the imaging. In order to find the optimum condition for the imaging, image calculations were made for the basic two compounds, $M$ and $Y$, since they include different kinds of the structural building unit from each other; $R$ and $T$ blocks, respectively. These calculations were based on the multi-slice method ${ }^{(14)}$ with a beam incidence parallel to the [1]0] axis in both structures. As the atomic scattering factors of the constituent atoms, those ${ }^{(15)}$ of the neutral state were used. The beam divergence $^{(16)}$, the chromatic aberration ${ }^{(17)}$ and the absorption effect $^{\dagger}$ were taken into consideration. The electron optical parameters used for $200 \mathrm{kV}$ high-resolution electron microscope are listed in Table 2.

The defocus positions at $60-65$ and $95-100$ $\mathrm{nm}$ almost correspond to the cases of $n=0$ and -1 in the aberration-free-focus positions $^{(18)}$ defined by $\left((1-2 n) C_{\mathrm{s}} \lambda\right)^{1 / 2}$, where $n$ is a negative integer number $(n \leq 0), C_{\mathrm{s}}$ the aberration constant of the objective lens and $\lambda$ the wave length. In the weak-phase-object approximation $^{(14)}$, a dark contrast is expected in the former case (Scherzer focus condition) at the position where the crystal potential is larger than average, while in the latter case a bright contrast is expected at the position.

Figure 2(a) shows the calculated throughfocus images of $M$ for thin specimen thicknesses. The atomic positions in $\mathbf{M}$ projected on (110) is schematically shown in (b). The image intensity is normalized to the difference between maximum and minimum intensities

$\dagger$ As the absorption potential, 1/10 of the projected potential was used. 


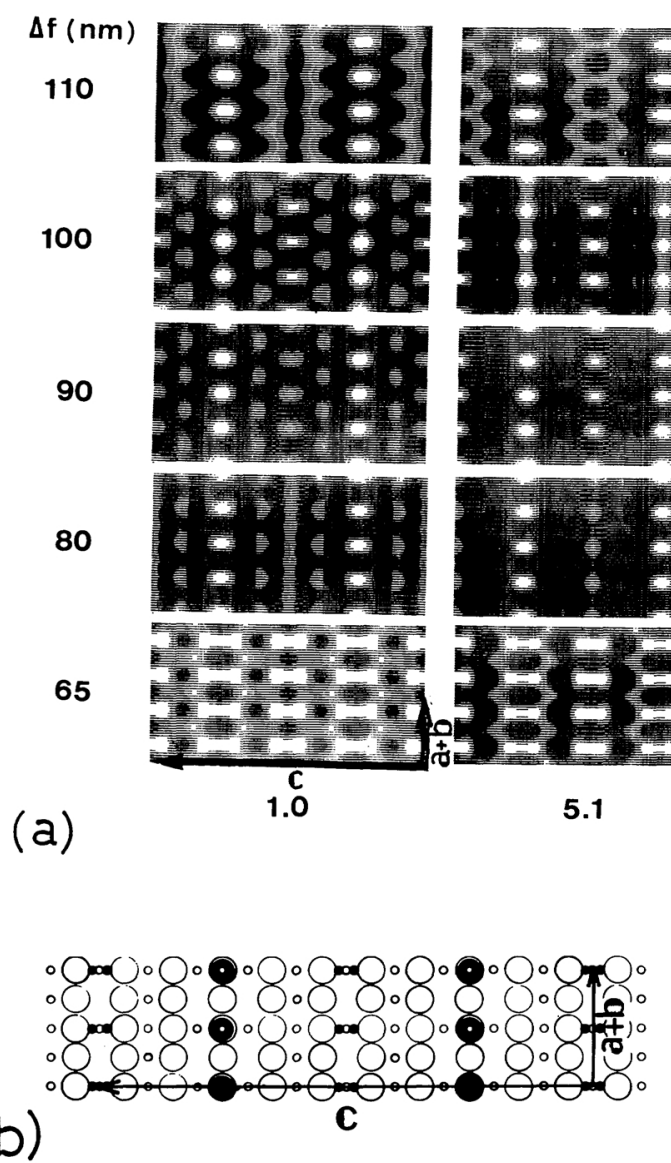

in each image. When the crystal thickness $t$ is very small, i.e. $t=1 \mathrm{~nm}$, and the weakphase-object approximation seems to be applicable, bright contrasts are seen at the barium atom positions in $\mathrm{R}$ blocks for $\Delta f=100 \mathrm{~nm}$, while dark contrasts are seen at those positions for $\Delta f=65 \mathrm{~nm}$. These results are consistent with the image modes mentioned above. When the crystal thickness becomes larger, however, contrast changes occur under both of the defocus conditions of $\Delta f=100$ and $65 \mathrm{~nm}$, and the positional correspondence between the barium atom positions and those of the bright or the dark contrasts becomes gradually poor.

On the other hand, under the focus condition $\Delta f=80-90 \mathrm{~nm}$, bright spot contrasts are also seen at the barium atom positions, and these contrasts become stronger (according to the difference between maximum and minimum intensities), as the thickness increases towards $15.3 \mathrm{~nm}$. Another important feature
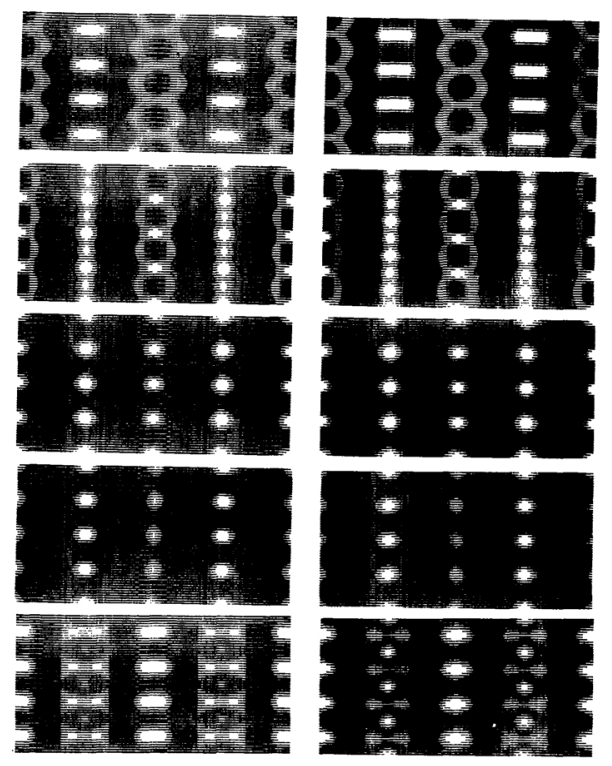

10.2

Fig. 2 (a) Calculated through-focus images of the $M$ compound for the defocus $65-110 \mathrm{~nm}$ (underfocus) and the thickness 1-15.3 nm. (b) Projection of the $M$ structure on the (110) plane. Large filled and open circles are barium and oxygen atoms, respectively, and interstitial iron atoms are indicated by small open (octahedral site) and filled (tetrahedral site) circles.

of the image under the defocus is that the middle positions of the $\mathrm{S}$ blocks, where iron atoms are densely located, are imaged as bright spots with moderate intensities. This indicates a possiblity of detecting $\mathrm{S}$ blocks also in the imaging. Therefore, we can conclude that the defocus condition near $80 \mathrm{~nm}$ would be an optimum one for the direct imaging of barium atom positions together with the positions of the middle of S blocks in a relatively wide thickness range. The reason for the appearance of bright spot contrasts at the correct positions of large projected potentials under the condition of $\Delta f \simeq 80 \mathrm{~nm}$, will be discussed in a later section with respect to the dynamical effect of the diffracted waves in the crystal.

Calculated through-focus images of Y compound for small crystal thicknesses show a similar tendency to those of $\mathbf{M}$ concerning the images at the metal atom positions. Defocus 

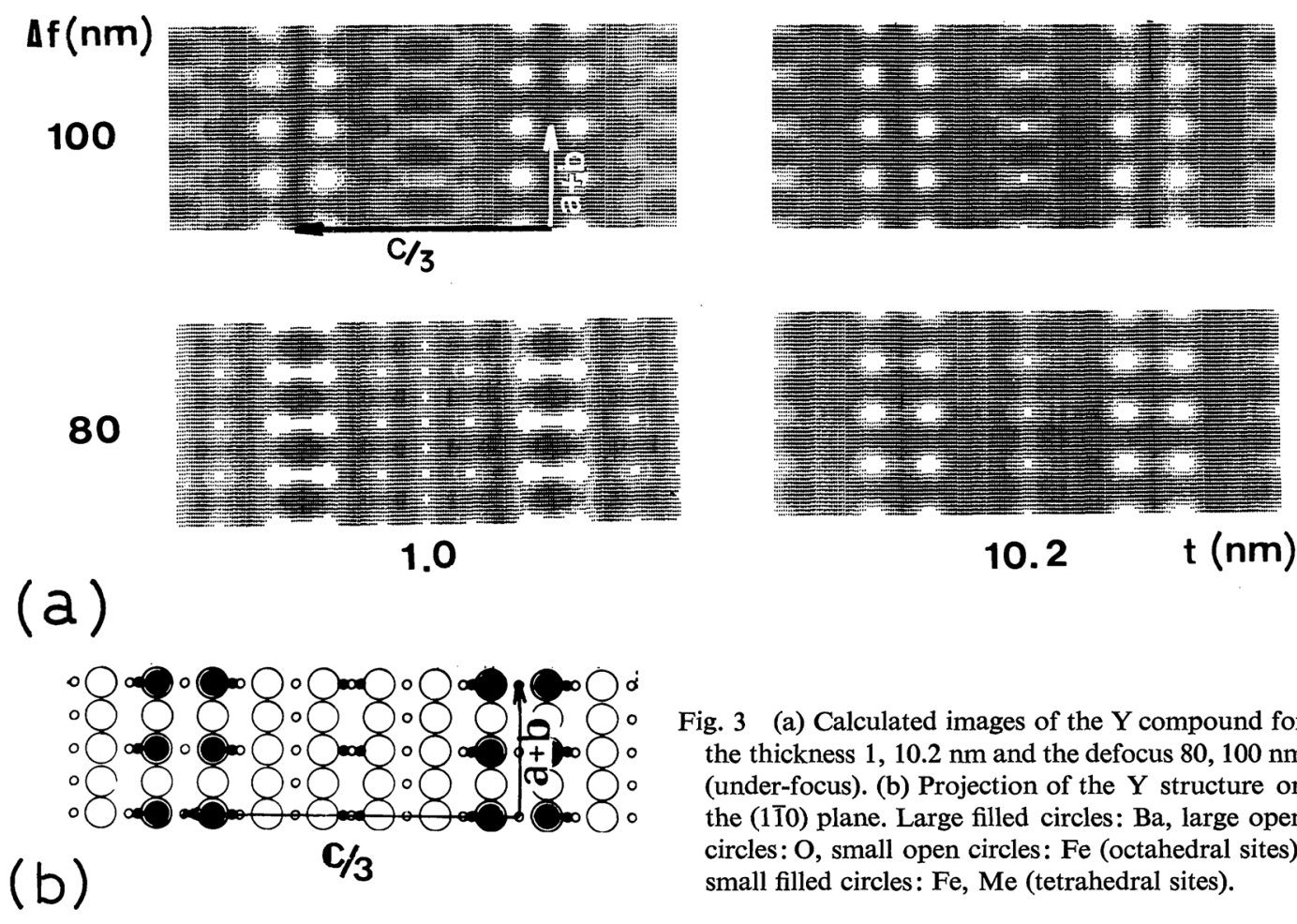

Fig. 3 (a) Calculated images of the $Y$ compound for the thickness $1,10.2 \mathrm{~nm}$ and the defocus $80,100 \mathrm{~nm}$ (under-focus). (b) Projection of the $Y$ structure on the (110) plane. Large filled circles: $\mathrm{Ba}$, large open circles: $\mathrm{O}$, small open circles: $\mathrm{Fe}$ (octahedral sites), small filled circles: Fe, Me (tetrahedral sites).

conditions near $80 \mathrm{~nm}$ under-focus give clear double bright spot rows normal to the $c$-axis almost corresponding to the barium positions in $\mathrm{T}$ blocks, while the condition $\Delta f=65 \mathrm{~nm}$ gives dark weak contrasts at those positions. Calculated examples are shown in Fig. 3(a) for $\Delta f=80$ and $100 \mathrm{~nm}$ under the thicknesses $t=1.0$ and $10.2 \mathrm{~nm}$. Different from the case in M, bright spots nearly corresponding to the barium atom positions in $\mathrm{T}$ blocks appear even for the case $\Delta f=100 \mathrm{~nm}$ under the condition of $t=10.2 \mathrm{~nm}$. In (b), (11) p) projection of atoms in $\mathrm{Y}$ is schematically shown. Metal atoms in the middle of $\mathrm{S}$ blocks are found to be also imaged as the same weak bright spots as they are in the case of $M$. Currect positional distances between neighboring barium atoms in the $\mathrm{T}$ blocks projected on the (110) plane are 0.294 and $0.293 \mathrm{~nm}$ along the directions of [110] and [001], respectively. An appreciable deviation of the bright spot images from the correct barium atom positions is found especially under the condition $\Delta f=100 \mathrm{~nm}, t=$ $1.0 \mathrm{~nm}$.

Image calculations assuming ionized states

of atoms were also made for both of the compounds $M$ and $Y$. Appreciable differences of the image contrasts between their neutral and ionized states were found only at the defocus $\Delta f=65 \mathrm{~nm}$ in both of the compounds.

\section{Experimental Procedure}

Specimens with compositions close to $\mathbf{M}$ and $\mathrm{Y}$ compounds were prepared by sintering mixed powders of high purity $\mathrm{BaCO}_{3}, \mathrm{ZnO}$ and $\alpha-\mathrm{Fe}_{2} \mathrm{O}_{3}$ at about $1473 \mathrm{~K}$ in argon atmosphere. Sintered specimens were crushed into thin flakes suitable for the electron microscopic observations.

High resolution observations were carried out by using JEM200-CX type electron microscope $(200 \mathrm{kV})$ with a high resolution top-entry goniometer stage. As a beam source, a pointed tungsten filament was used. In every crystal, images were taken under the axial illumination condition along the zone axis of [110] at a magnification of $2.7 \times 10^{5}$ times. A defocussing condition of $\Delta f=60-90 \mathrm{~nm}$ under-focus was used in the imaging throughout the observa- 
tion. The amount of defocus was estimated based on the Fresnel fringe method ${ }^{(19)}$.

\section{Observations}

\section{Confirmation of the imaging conditions for $\mathrm{Ba}$ atom positions}

Figure 4 shows an example of the image of $M$ compound taken under the focus condition near $80 \mathrm{~nm}$ under-focus. Strong bright spot rows normal to the $c$-axis are observed with an interval of about $0.3 \mathrm{~nm}$. The distance between the periodic rows along the $c$-axis equals to a half of the axial length of $c$ for $\mathbf{M}$. Weak bright spot contrasts between these strong bright spot rows are also observed. Near the specimen edge, an image contrast change is seen which is due to a small local change in the defocus. The image mode of the bright spots shown is in good agreement with the calculated ones in Fig. 2(a) for $\Delta f=80 \mathrm{~nm}$, $t=5.1-15.3 \mathrm{~nm}$. It is reasonable, therefore, to

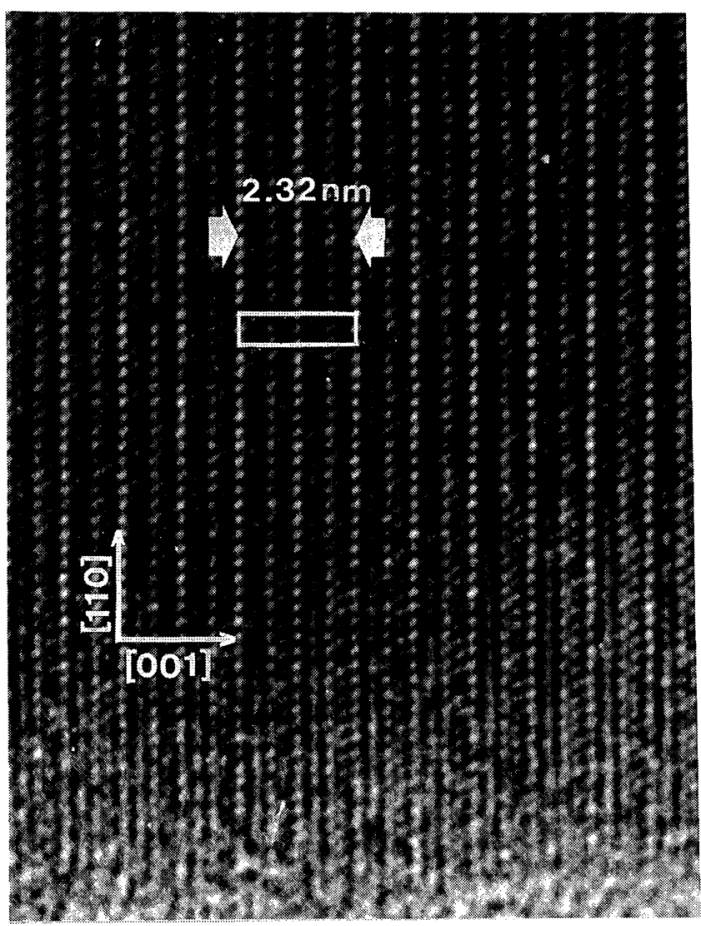

Fig. 4 High resolution image of the $\mathbf{M}$ compound taken under the defocus $\Delta f \sim 80 \mathrm{~nm}$ (under-focus). The beam incidence is along the [110] direction. The projected unit cell size is indicated by a rectangle.

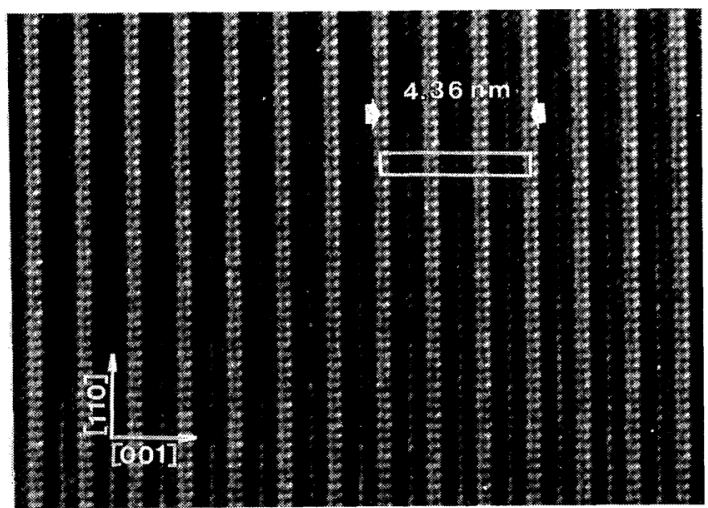

Fig. 5 High resolution image of the $Y$ compound taken under the defocus $\Delta f \sim 80 \mathrm{~nm}$ with the beam incidence of $[1 \overline{1} 0]$. A rectangle indicates the unit cell size.

think that barium atom positions in $\mathrm{R}$ blocks are imaged together with iron atom positions in $\mathrm{S}$ blocks as strong and weak bright spots, respectively, under the focus condition near $\Delta f=80 \mathrm{~nm}$.

Figure 5 shows an enlarged image of $Y$ compound in a thin area of the specimen also taken under the focus condition near $80 \mathrm{~nm}$. Paired bright spot rows normal to the $c$-axis are imaged, arranging periodically along the [001] direction with a spacing of $1 / 3$ of the axial length of the $c$-axis. Spacings between the neighboring bright spots are both about 0.3 $\mathrm{nm}$ along normal and parallel directions to the $c$-axis. Between the two parallel rows of paired bright spots, a single bright spot row with weak intensity is observed. All the positions and the contrasts of the bright spots mentioned above are in good agreement with those calculated in Fig. 3. It has been confirmed that the barium atom positions in $\mathrm{T}$ blocks are also visible as bright spots under the present observation condition.

\section{Compositional faults}

Since there is a good correspondence between the calculated and the observed images of barium atom positions in $\mathrm{R}$ and $\mathrm{T}$ blocks in addition to the metal atom positions in $\mathrm{S}$ blocks under the condition of $\Delta f \simeq 80 \mathrm{~nm}$, it is suggested that the direct structure analysis in hexagonal ferrites is possible including the 


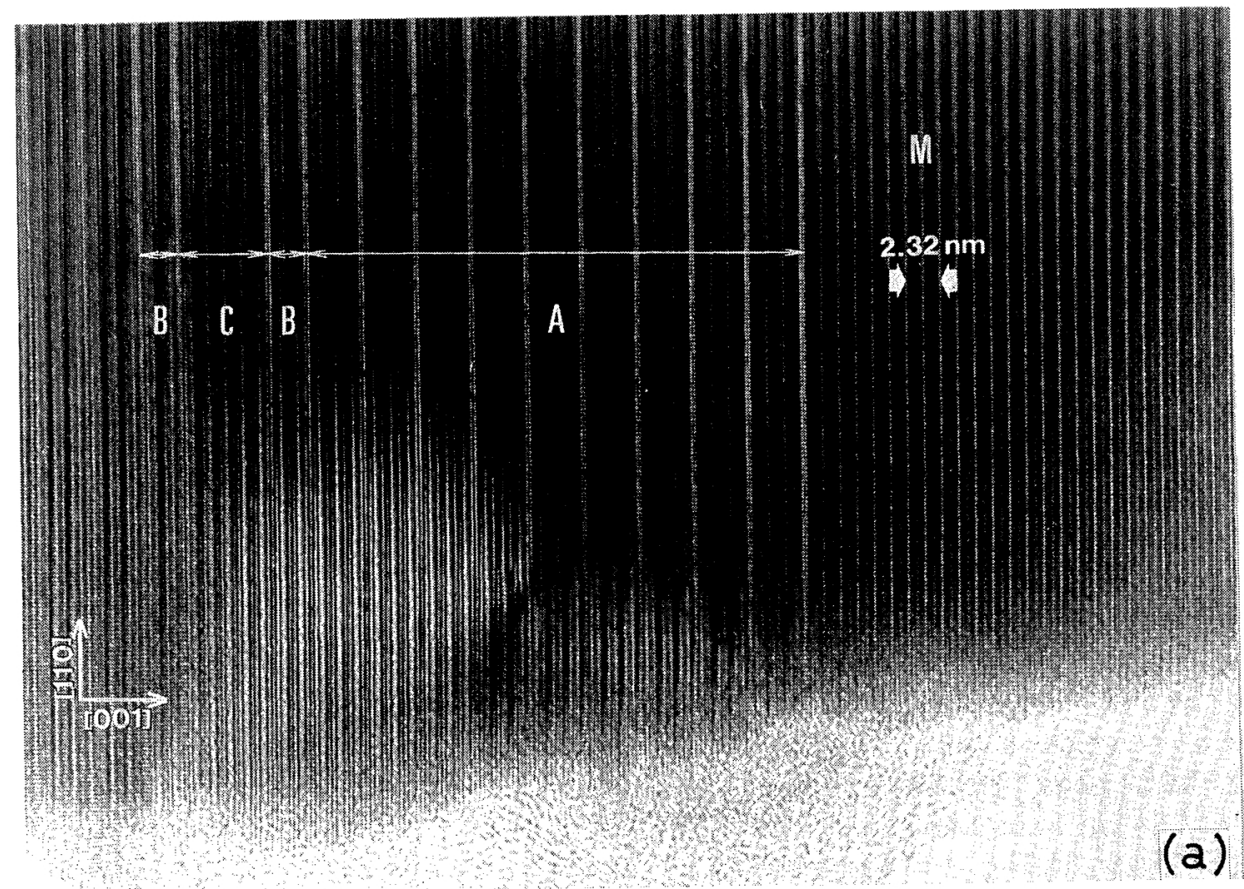

Fig. 6 (a) High resolution image showing compositional faults introduced in the $\mathrm{M}$ compound. (b) Enlarged image of the structure lettered by $\mathrm{A}$ intergrown in the $\mathbf{M}$ structure.
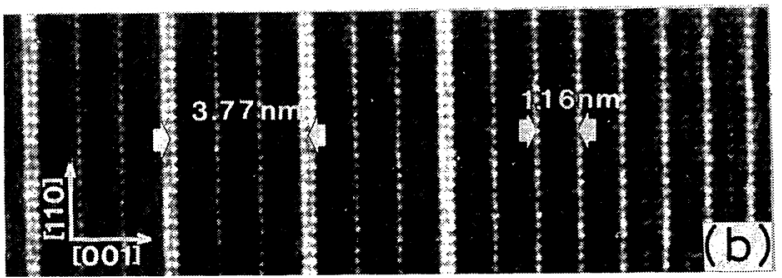

analysis of the compositional faults introduced by the microsyntactic intergrowth. This can easily be done by the examination of the sequence mode of the $\mathrm{R}, \mathrm{T}$ and $\mathrm{S}$ blocks represented, respectively, by the single and paired bright spot rows with high contrasts and the single bright spot row with a low contrast.

Figure 6(a) shows an image where a structural modulation occurs in the $\mathbf{M}$ compound, which was taken under the conditions of $\Delta f \simeq$ $80 \mathrm{~nm}$ and the beam incidence [110] of $M$. With similar image contrasts, three kinds of periods letterd by A, B and C are seen which are different from the period of $\mathrm{M}$ seen on the right side of the image. An enlarged image of the area $A$ joining to the $M$ structure is shown in Fig. 6(b). Normal to the [001] direction of $M$, paired bright spot chains with the pair distance of about $0.3 \mathrm{~nm}$ are periodically arranged with a spacing of $3.8 \mathrm{~nm}$. Between these periodic paired chains, two parallel chains of bright spots are seen with a positionally in-phase relation to those of the $M$ structure. Based on the image codes of $\mathrm{R}, \mathrm{T}$ and $\mathrm{S}$ blocks under the defocus $\Delta f \simeq 80 \mathrm{~nm}$, it is immediately found that the structure of the area $\mathrm{A}$ is composed of block sequences of TSRSRS. Similarly, block sequences in the areas B and C are found to be TSRS and TSRSRSRS, respectively, along the $c$-axis direction of $M$. The intergrowth phase in the area $\mathrm{A}$ is identified to be the $U$ compound with a rhombohedral symmetry represented by the stacking notation (TSRSRS) ${ }_{3}$ (see Table 1), since no hexagonal symmetry can be expected from the block sequence. Structures in the areas $\mathrm{B}$ and $\mathrm{C}$ are identified to be isolated $1 / 2$ periods of the hexagonal $Z$ and $M_{3} Y$ compounds, respectively. It seems that these isolated structural units are intergrown in order to compensate 
the composition fluctuations in those areas.

\section{Discussion}

In this study, it was found that the focusing at $\Delta f \simeq 80 \mathrm{~nm}$ gives an optimum focus condition for the direct imaging of barium atom positions in both $\mathrm{R}$ and $\mathrm{T}$ blocks in the relatively wide thickness range, in spite of its deviation from the aberration-free-focus conditions. In order to interprete this, amplitudes and phases of several important reflections from the $\mathrm{M}$ compound were examined as a function of the specimen thickness and the defocus.

In the imaging, not only the amplitudes of the diffracted beams but also their phases play an important role on the intensity distribution of the image. A phase change of a diffracted beam occurs by the defocus and the spherical aberration of the objective lens and also by the dynamical interactions between the beam and other beams excited in the crystal. Amplitudes and phases of the reflections $0,0,2 n(n \leq 5)$ and $1,1,2 m(m \leq 3)$ were calculated, where $n$ and $m$ were integer numbers. These reflections pass through the objective aperture and have enough amplitudes for the imaging after filtered by the envelope functions. An example of electron diffraction patterns from the $M$ compound used for the high resolution imaging is shown in Fig. 7.

The amplitude effective for the imaging can be written as

$$
A_{h}=\left[\psi_{h}(t) C_{h} / \psi_{0}(t)\right] \exp \left[i\left(\varphi_{h}(t)-\varphi_{0}(t)+\chi_{h}\right)\right],
$$

where $\psi_{0}(t)$ and $\psi_{h}(t)$ are the wave amplitudes of the direct and the diffracted beams at the crystal thickness of $t, \varphi_{0}(t)$ and $\varphi_{h}(t)$ their respective phases, $C_{h}$ the spread function composed of the envelope and the aperture functions effective for the high spacial frequency range and $\chi_{h}$ the phase factor used in the phase contrast transfer function. The phase term

$$
\phi_{h}(t)=\varphi_{h}(t)-\varphi_{0}(t)+\chi_{h},
$$

is an effective phase change responsible for the imaging. Figure 8 shows the calculated absolute amplitudes $\left|A_{h}\right| \quad\left(=\psi_{h}(t) C_{h} / \psi_{0}(t)\right)$ for the 0 ,

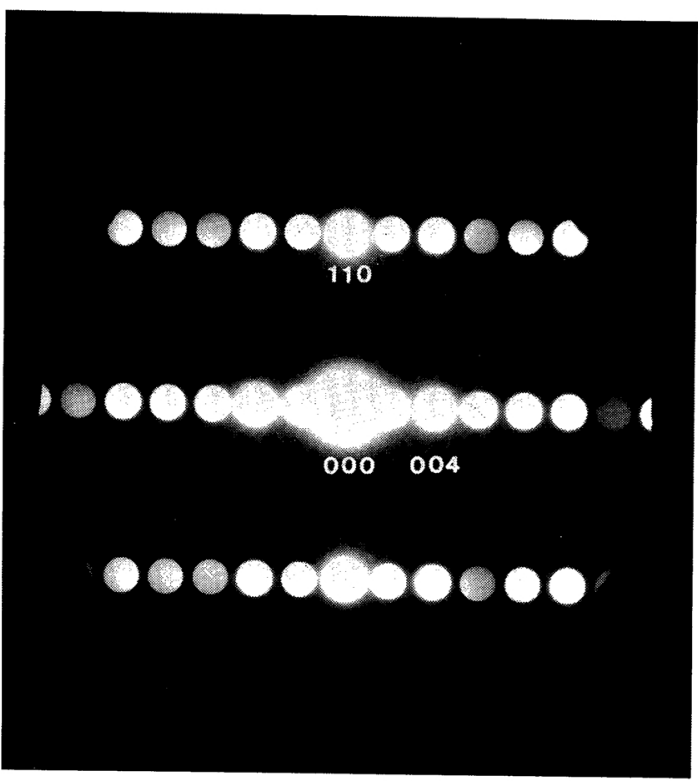

Fig. 7 Electron diffraction pattern of the $M$ compound used for the high resolution imaging, whose field is limited by the objective aperture.

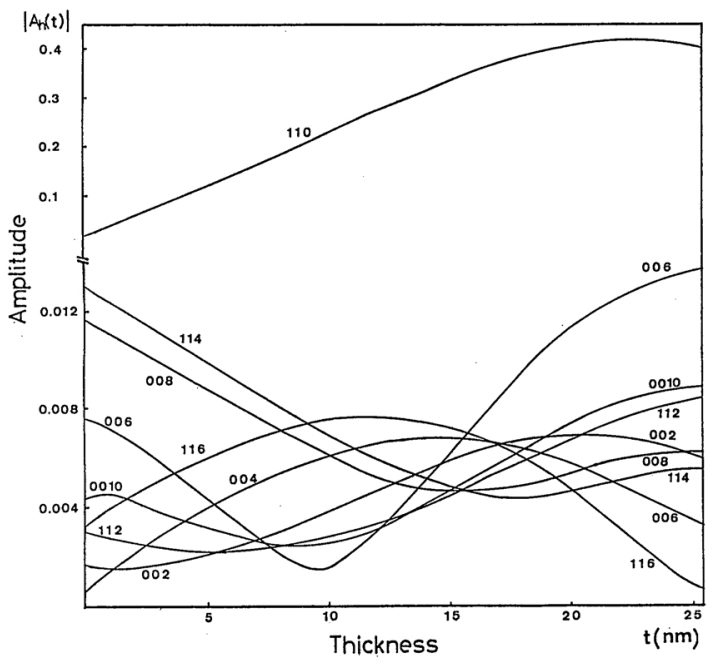

Fig. 8 Thickness dependence of the absolute amplitudes $\left|A_{\mathrm{h}}(t)\right|$ of the $0,0,2 n$ and $1,1,2 m$ reflections for the $\mathrm{M}$ structure.

$0,2 n$ and the $1,1,2 m$ reflections. The phase changes $\phi_{h}(t)$ of these reflections relative to the thickness are shown in Fig. 9 for $\Delta f=80 \mathrm{~nm}$.

According to Fig. 9, most of the important reflections for the imaging of high crystal potential projections in $\mathrm{R}$ and $\mathrm{S}$ blocks have phases between $0 \lesssim \phi_{h}(t) \lesssim-\pi / 2$ in the crys- 


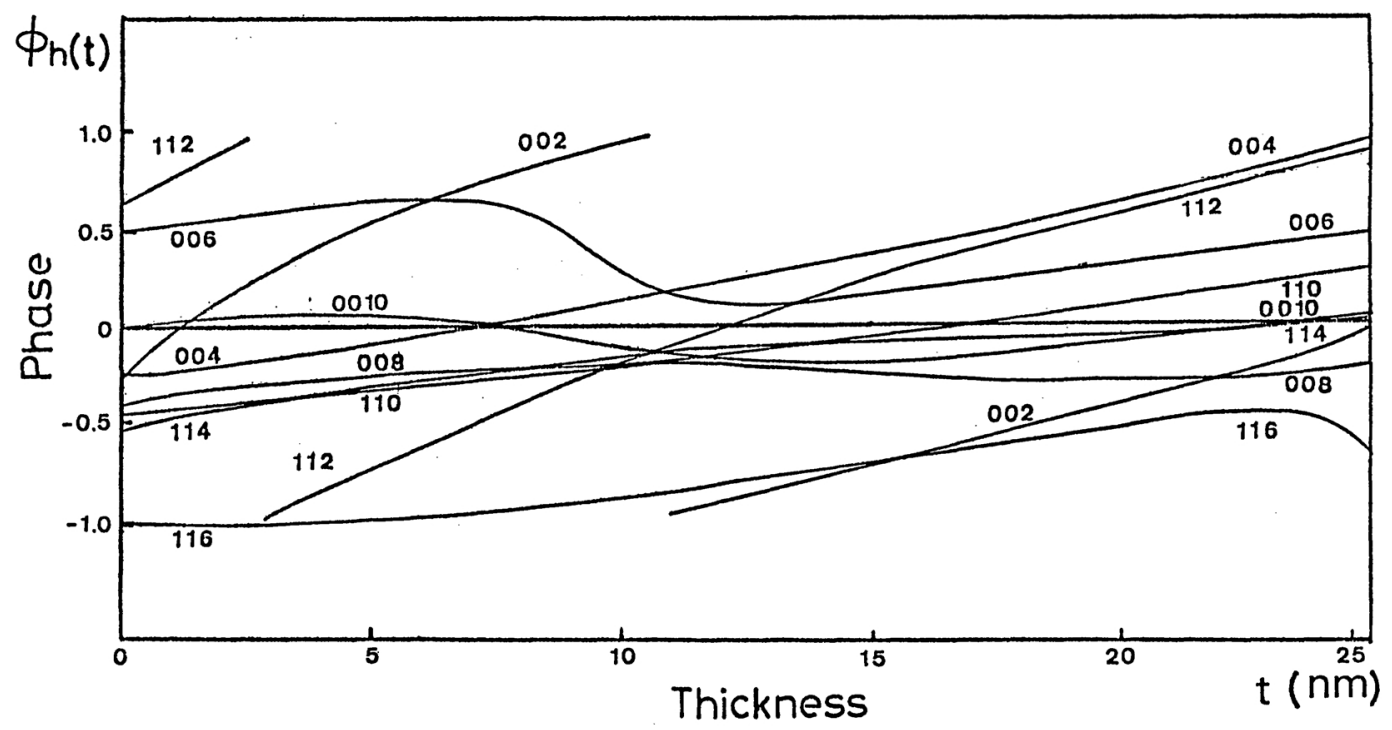

Fig. 9 Thickness dependence of the phase changes $\phi_{h}(t)$ of the $0,0,2 n$ and $1,1,2 m$ reflections for the M structure. The vertical scale is in units of $\pi$.

tal thickness range $0 \lesssim t \lesssim 17 \mathrm{~nm}$. This indicates the formation of the bright spot contrasts at the positions of high potential projections in the thickness range. At the thickness near $10 \mathrm{~nm}$, in-phase relations are seen among the reflections 110, 112, 114,008 and 0010 which have larger real part values of their amplitudes than the imaginary part values. At the thickness $t \sim$ $10 \mathrm{~nm}$, an out of phase relation is seen between the reflections 004 and 116 which have a phase difference of about $\pi$. These two reflections are thought to have minor contributions to the imaging, since their amplitudes $A_{h}(t)$ cancell each other due to almost the same magnitudes of their values of $\left|A_{h}(t)\right|$ (Fig. 8). A similar relation is also found between 002 and 006 reflections in the thickness region. The above phase relations indicate that the inphase reflections $110,112,114,008$ and 0010 contribute predominantly to the formation of the bright spot contrasts. This is the reason why a clear interpretable bright spot image appears in a rather thicker part of the specimen. In the smaller thickness range, $t \lesssim 7 \mathrm{~nm}, 004$, 110,114 and 008 reflections become predominant for the image formation. But in this case, a weaker image contrast is expected than in the case of the crystal thickness $t \gtrsim 10 \mathrm{~nm}$, since 110,114 and 008 reflections contribute to the positive image contrast mainly by the imaginary parts of their amplitudes. As the thickness becomes larger, the reflections tend to have less chances to be in in-phase relations with one another.

According to the phase calculations made for $\Delta f=100 \mathrm{~nm}$ underfocus, the phase relations responsible for the bright spot image which correctly shows the barium atom positions are found only in the small thickness region of $t \lesssim 3 \mathrm{~nm}$, in good accordance with the result of the image calculation in Fig. 2(a).

From the calculated retults of amplitudes and phases of the reflections of the $\mathrm{M}$ compound, it is concluded that the meaningful image reproducing the projected potential can be obtained when several important reflections have in-phase relations with relatively larger real part values for their amplitudes compared with the imaginary parts, even if the relation $\phi_{h}(t)=2 n \pi$ is not satisfied. This conclusion can be applicable also in the case of the $\mathrm{Y}$ compound. In this case, several reflections in the high spacial frequency range like $0012,0015,0018,110,113$ and 116 must have in-phase relations to form the paired bright spot rows separated by $0.29 \mathrm{~nm}$. Since such an interpretable image as calculated 
under the condition of $\Delta f=80 \mathrm{~nm}, t=10.2$ $\mathrm{nm}$ is also reproduced under $\Delta f=100 \mathrm{~nm}$ and $t=10.2 \mathrm{~nm}$ (Fig. 3(a)), the above phase relation seems to stand for also in the latter case.

Hexagonal ferrites are structurally composed of the oxygen frameworks in which oxygen atoms are periodically replaced by the barium atoms. In this sense, we have a similarity between the structures of hexagonal ferrites and the long period superstructure of alloys. According to the examination of the details of the structure factors for $M$ and $Y$ compounds, however, reflections from interstitial metal atoms contribute to the structure factors fairly largely, and most of them have different phase relations to the barium atom reflections. Therefore, it is suggested that the term "superstructure image" is not suitable for the bright spot images observed in the present study.

\section{Acknowledgment}

This work was partly supported by the Grant-in-Aid for the Scientific Research from the Ministry of Education, Science and Culture.

\section{REFERENCES}

(1) J. Smit and H. P. J. Wijn: Ferrites, John Wiley and Sons, Inc., New York, (1959), p. 191.

(2) J. A. Kohn and D. W. Eckart: Z. Kristallogr.,
119 (1964), 454.

(3) J. A. Kohn, D. W. Eckart and Charles F. Cook, Jr.: Science, 172 (1971), 519.

(4) J. Van Landuyt, S. Amelinckx, J. A. Kohn and D. W. Eckart: J. Solid State Chem., 9 (1974), 103.

(5) Y. Hirotsu and H. Sato: J. Solid State Chem., 26 (1978), 1.

(6) Y. Hirotsu, H. Sato, Y. C. Tang and S. Nagakura: Ferrites, Proc. 3rd Inter. Conf. on Ferrites, Kyoto, (1980), p. 345.

(7) F. J. A. Den Broeder: J. Solid State Chem., 37 (1981), 362.

(8) M. Hirabayashi, K. Hiraga and D. Shindo: J. Appl. Cryst., 14 (1981), 169.

(9) K. Hiraga, D. Shindo and M. Hirabayashi: J. Appl. Cryst., 14 (1981), 185.

(10) O. Terasaki and D. Watanabe: J. Appl. Cryst., 15 (1982), 282.

(11) N. Kuwano, M. Toki, N. Tanaka and T. Eguchi: Proc. 6th Int. Conf. High Voltage Electron Microscopy, Vol. 4, Antwerp, (1980), p. 166.

(12) D. Shindo: Acta Cryst., A38 (1982), 310.

(13) O. Scherzer: J. Appl. Phys., 20 (1949), 20.

(14) J. M. Cowley: Diffraction Physics, North-Holland Pub. Inc., Amsterdam, (1975).

(15) P. A. Doyle and P. S. Turner: Acta Cryst., A24 (1968), 390.

(16) J. Frank: Optik, 38 (1973), 519.

(17) P. L. Fejes: Acta Cryst., A33 (1977), 109.

(18) F. Thon: Z. Naturforsch., 21a (1966), 476.

(19) L. A. Bursill and A. R. Wilson: Acta Cryst., A33 (1977), 672. 